Research Article

\title{
Tunable Nanodielectric Composites
}

\author{
Daniel Qi Tan, ${ }^{1}$ Yang Cao, ${ }^{1,2}$ Xiaomei Fang, ${ }^{1}$ and Patricia C. Irwin ${ }^{1}$ \\ ${ }^{1}$ GE Global Research Center, Niskayuna, NY 12309, USA \\ ${ }^{2}$ Department of Electrical and Computer Engineering, University of Connecticut, Storrs, CT 06269, USA
}

Correspondence should be addressed to Daniel Qi Tan; tan@ge.com

Received 12 November 2013; Revised 22 January 2014; Accepted 12 February 2014; Published 25 March 2014

Academic Editor: A. G. Barbosa de Lima

Copyright (C) 2014 Daniel Qi Tan et al. This is an open access article distributed under the Creative Commons Attribution License, which permits unrestricted use, distribution, and reproduction in any medium, provided the original work is properly cited.

\begin{abstract}
This paper presents a progress update with the development of nanodielectric composites with electric field tunability for various high energy, high power electrical applications. It is demonstrated that nonlinear electrical/dielectric properties can be achieved via the nanostructure and interface engineering. A high level summary was given on the progress achieved as well as challenges remaining in nanodielectric engineering towards high energy density capacitors for energy storage and conversion, nonlinear dielectrics for tunable device, and high voltage varistor for surge suppression.
\end{abstract}

\section{Introduction}

The rapid expansion of renewable energy applications demands higher efficiency and higher density energy storage and energy conversion systems $[1,2]$. Various DC-AC, AC-AC conversions are needed for solar and wind farms, while primary and secondary electrochemical devices are in need for transportation and telecommunication applications. Advanced devices and components are critical enablers for these emerging applications. As an example, in a typical electrical converter/inverter, state-of-art DC-link capacitors take up about $30 \%$ total volume and weight. In addition, the wide usage of renewable energy resources such as wind and solar posts challenges in grid stability. Advanced passive and active devices will be needed for future grid for harmonic filtration, static/dynamic volt-ampere reactive (VAR) compensation, transient suppression, and so forth $[3,4]$.

All these advanced apparatus and future electric power infrastructure rely on the breakthroughs of material engineering and better understanding of the device physics [1]. For instance, the development of high dielectric constant materials and the understanding of the behavior of dielectric materials under extremely high electric fields are critical for energy storage applications [5]. In addition, better understanding and control of charge transport cross interfaces are crucial for nonlinear devices. The advance in nanotechnology offers a unique opportunity to engineer materials with controlled microstructures so that desirable electrical properties can be greatly enhanced $[1,6]$. On the other hand, dielectric response of materials can be utilized to facilitate the formation of nanostructures [7]. In this paper, progress updates are given as exemplary cases on the development of nanoenabled composite materials for high energy density capacitors for energy storage and conversion, field tunable nonlinear dielectrics, and miniaturized varistors for high voltage/high current transient suppression.

\section{Experimental}

In this investigation, polyetherimide (PEI), silicone, cyanoethyl cellulose, polyimide, and poly(vinylidene difluoride) were used as the polymer matrices in the investigation. Nanoparticles of interest include oxides of silicon, niobium, aluminum, zinc, bismuth, antimony, cobalt, titanium, and barium titanate and lead zirconates, with the particle size in the range of 10 to $100 \mathrm{~nm}$. Polymer nanocomposites were prepared by first dissolving a polymer resin in a solvent at room temperature with a magnetic stirrer and then mixing with nanoparticles of $2-50 \mathrm{vol} \%$ in a high-energy sonicator (Sonics \& Materials, Inc. Newtown, CT). The films were solvent cast onto a glass slide and dried at $100^{\circ} \mathrm{C}$ for two hours followed by a vacuum dry at $120^{\circ} \mathrm{C}$ to $150^{\circ} \mathrm{C}$ overnight. Ceramic composites were formulated with at least $85 \mathrm{~mol} \%$ 
$\mathrm{ZnO}$ and other oxide additives. A conventional mixed oxide process was used for powder processing. Green compacts of $1^{\prime \prime}$ diameter were pressed using a uniaxial hydraulic press and then sintered at temperatures of $850-1000^{\circ} \mathrm{C}$.

DC breakdown test was conducted following ASTM D149 (method A) using a ball-plane electrode configuration. The sample was immersed into insulation oil and DC voltage was applied at a ramp rate of $500 \mathrm{~V} / \mathrm{s}$ until the sample failed. Dielectric responses were measured using a broadband dielectric spectrometer from Novacontrol GmbH. Scanning electron microscopy (SEM) imaging was done using a Zeiss Supra 55VP (Carl Zeiss AG, Oberkochen, Germany). Transmission electron microscopy (TEM) imaging was taken using a FEI Tecnai F20 transmission electron microscope.

\section{Results and Discussions}

High energy density capacitor dielectrics, field tunable composite dielectrics, and metal oxide varistors (MOVs) are taken as examples in this section to demonstrate the achievable electrical/dielectric properties via the nanodielectric and interface engineering to fulfill the aforementioned development needs.

3.1. Nanofilled Polymer Film for Capacitors. Capacitors represent a big family of non-Faradic energy storage components used in applications ranging from decoupling in circuit board to series capacitive compensation banks at power transmission class. The state-of-art polymeric film capacitors are based primarily on low permittivity polymers such as polypropylene or polyester. One natural approach for higher energy density capacitor is the synthesis of higher permittivity composites with higher dielectric strength by incorporating ceramic fillers into polymer matrix. This is because the energy density of a film capacitor takes the simple form of $1 / 2 \varepsilon_{o} K E^{2}$, where $\mathrm{K}$ is the dielectric constant and $E$ is the dielectric strength of the polymer film.

Although the dielectric constant can be increased to some extent at higher particle loading level, increasing the dielectric strength remains a great challenge. Instead, the addition of particles exceeding $10 \mathrm{wt} \%$ usually results in the considerable loss of dielectric strength of the polymer. Figure 1 is a graphical comparison of the DC breakdown strength for various nanofilled PEI films. With more conductive fillers, lower breakdown strength is not surprising. However, the insulating ceramic fillers of higher breakdown strength do not improve the electrical field endurance of the nanocomposites.

With decreasing particle size, interfacial fraction increases and the particle-polymer interface adhesion also becomes critical. Poor particle dispersion and interaction with polymer matrix is shown in Figures 2(a) and 2(b). It can be seen that both dry alumina and silica particles are agglomerated and remain to be in poor dispersion even after mixing using a high energy ultrasonic vibration. The high surface energy and new chemistry on the nanoparticle surface tend to bond the particles together tightly.

In order to minimize the particle surface effect, colloidal particles that were already well dispersed in a solvent were

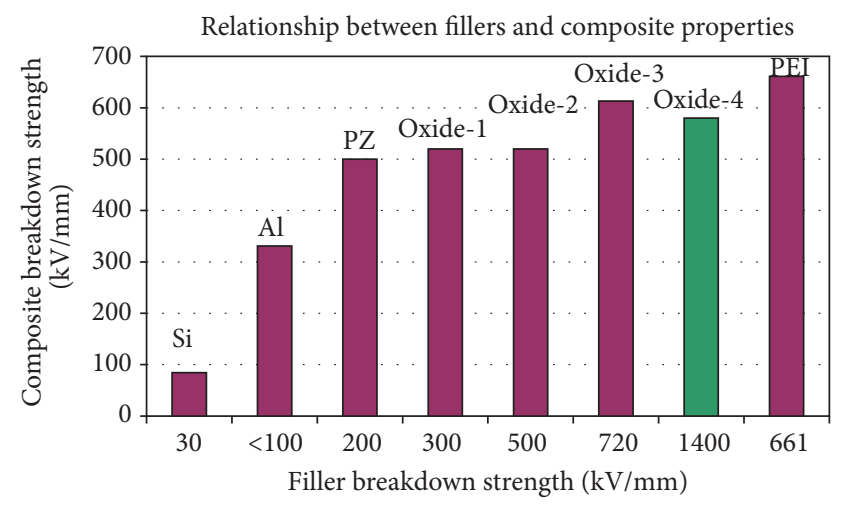

FIgURE 1: The breakdown strength of PEI nanocomposites with various fillers of about $5 \mathrm{wt} \%$ loading. $\mathrm{PZ}$ stands for lead zirconate. All polymer films were processed using a solvent cast.

procured and mixed with a polymer resin in the wet state. Figure 2(c) shows the image of precursor $\mathrm{SiO}_{2}$ particles. After performing in situ polymerization of the polyamic acid, a polymer nanocomposite containing 5 vol\% $\mathrm{SiO}_{2}$ nanoparticles was synthesized. Great particle dispersion was achieved as shown in Figure 2(d). In order to achieve high energy density in a nanodielectric composite, a compromise between increase in dielectric constant and decrease of dielectric strength when doing nanodielectric engineering appears to be the direction.

3.2. Field Tunable Nonlinear Composites. The success of nanodielectric engineering depends on several factors, including (1) higher dielectric constant polymer matrix, (2) high permittivity ceramic fillers with low (hysteresis) loss, (3) proper dispersion, and (4) good interfacing between filler and polymer matrix $[8,9]$. This becomes even more critical when the particle polarity is increased resulting in higher dielectric constant. Among the polar ceramic materials are ferroelectric (FE) and antiferroelectric (AFE) particles, whose impact on dielectric properties and dispersion are still not well understood [9]. Figure 3 shows the increase of the effective dielectric constant of nanocomposites from addition of particles. Typically, the dielectric constant contrast between high- $k$ ceramic filler and polymer matrix is so high that the electric field concentration in polymer phase will reduce the breakdown strength of the nanocomposites. In addition, high dielectric constant contrast also leads to low electric field penetration into the particles and thus the reduced overall energy storage density in the composites.

In order to enable the nonlinear tunability of polymer, we leveraged ferroelectric $\mathrm{BaTiO}_{3}$ and antiferroelectric lead zirconate nanoparticles (nPZ). The electric filed tunable behavior of the composites was further studied under high electric fields. The nonlinear dependence of the dielectric constant of the polymer composites was fully exhibited as the electric field is increased exceeding the coercive field of lead zirconate (nPZ) as shown in Figure 4. The higher the electric field and loading fraction of particles, the higher the dielectric constant was reached. The field tunable behavior of composites enabled by the polar particles can be applicable for 


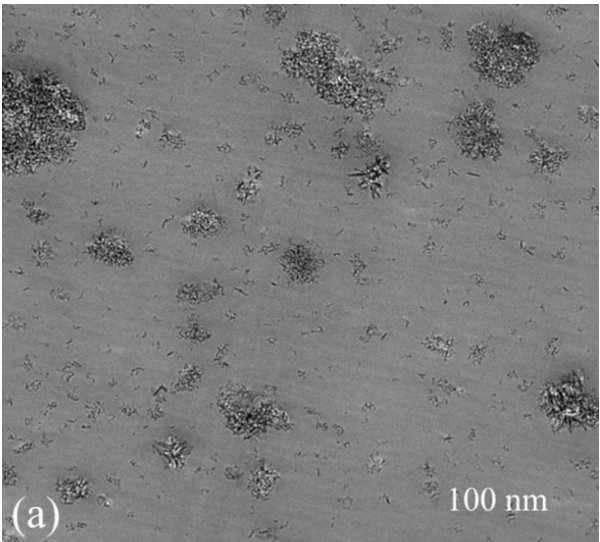

(a)

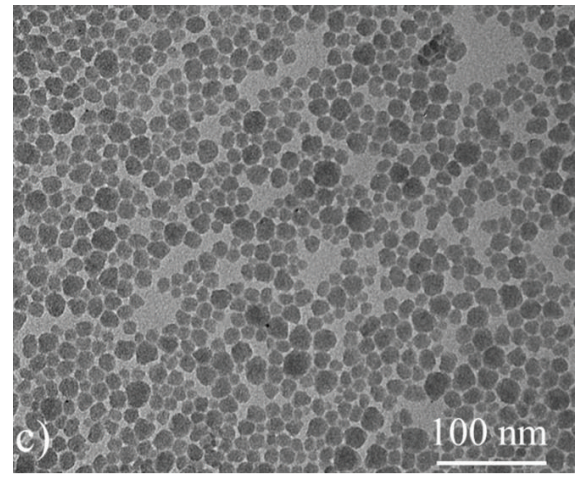

(c)

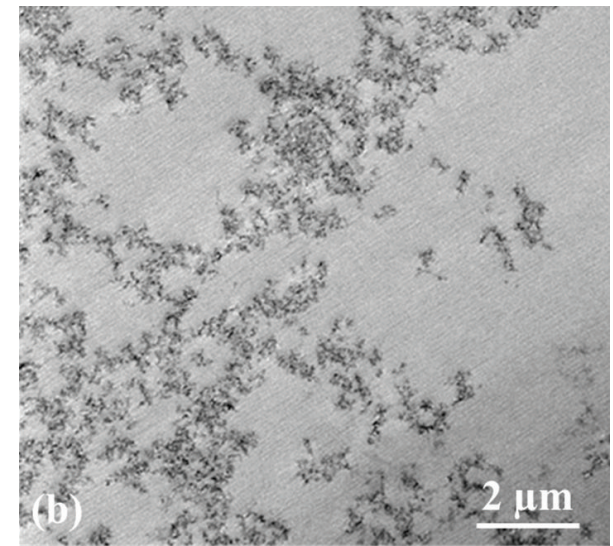

(b)

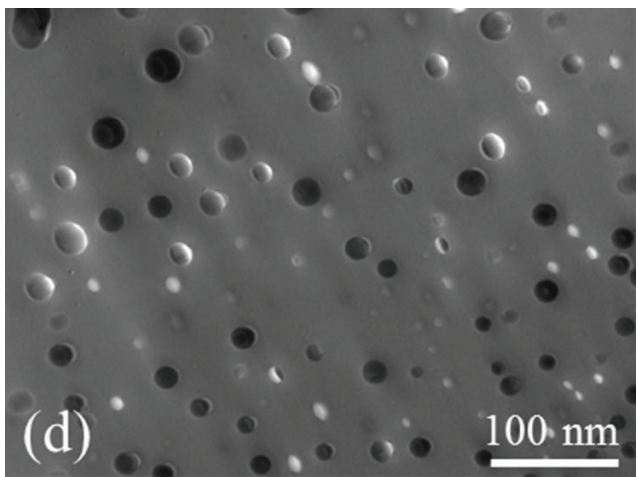

(d)

FIGURE 2: TEM image of 5 vol\% nanoparticles dispersing in polymer films. (a) Dry $\mathrm{Al}_{2} \mathrm{O}_{3}$, (b) dry silica, (c) colloidal $\mathrm{SiO}_{2}$, and (d) colloidal $\mathrm{SiO}_{2}$ in a polyimide.

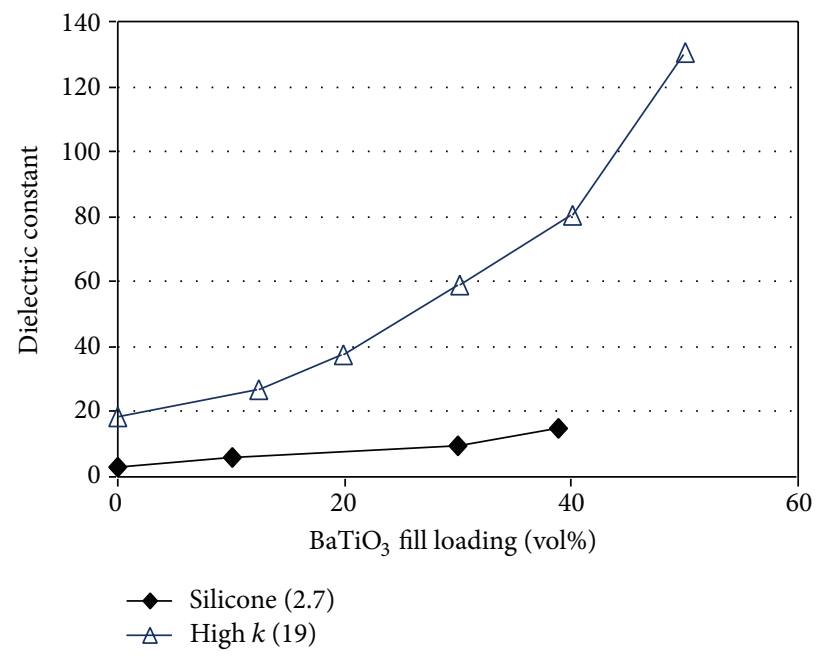

FIgURE 3: The dielectric constant of nanocomposites as a function of volume pertentage loading of $\mathrm{BaTiO}_{3}$ nanoparticle in silicone and cyanoethyl cellulose with dielectric constant of 2.7 and 19 , respectively. 


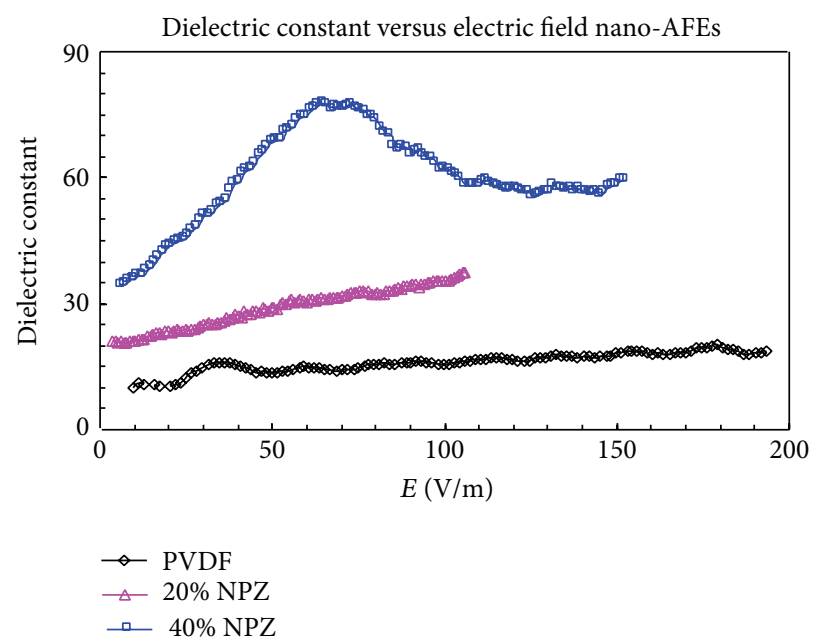

Figure 4: Room temperature dielectric constant under an increased electric field. Values were obtained from the hysteresis loop measurements of a PVDF polymer containing nPZ particles $(10 \mathrm{~Hz})$.

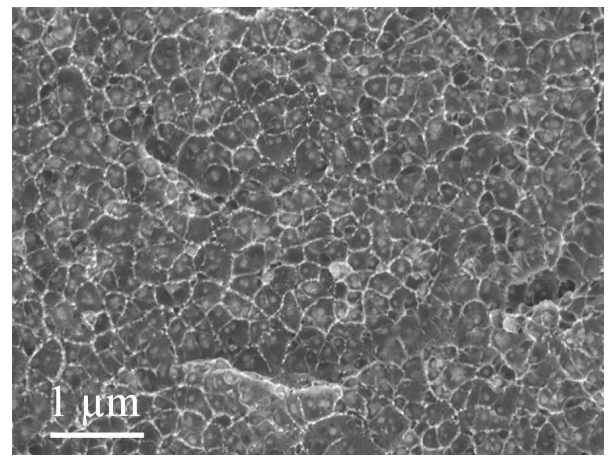

FIGURE 5: TEM image of polyimide containing $15 \mathrm{vol} \% 40 \mathrm{~nm} \mathrm{BaTiO}_{3}$ nanoparticles through in situ polymerization of 2,2-bis[4-(3,4dicarboxyphenoxy) phenyl]propane dianhydride (BPADA) with $4,4^{\prime}$-oxydianiline (ODA). Film was solution cast and dried and completely imidized at elevated temperatures.

various devices such as antenna and phase shifters requiring frequency tunability. A breakdown strength of $>300 \mathrm{kV} / \mathrm{mm}$ was also achieved in the polar nanodielectric composites, rendering an energy density of up to $15 \mathrm{~J} / \mathrm{cm}^{3}$.

TEM imaging shows the great dispersion and particle distribution in the polymer achieved using properly processed particles and mixing method (Figure 5). The fractured crosssection image of the film reveals only cohesive fracture within polymer rather than adhesive failure at interface indicating a strong polymer-particle bonding.

3.3. Nanoenabled Nonlinear Resistor (Varistors). High capacity, high density power system may be subject to high voltage transients and surge protection devices are widely used. Compared with other protection devices such as TVS, metal oxide varistor (MOV) as an example of ceramic-based nonlinear dielectric material at low voltage provides a good combination of high voltage scalability, peak current carrying capability, and fast response speed [10]. Nanoenabled dielectric composites were also studied in both metal oxide varistors and polymer based varistors. MOV is made of bulk ceramic containing predominate grain-boundary barrier junctions. However, the underlying oxide processing requires high sintering temperatures $\left(1000-1300^{\circ} \mathrm{C}\right)$ for densification. As a result, compositional and microstructural heterogeneity and large average grain sizes (typically from a few microns to tens of microns, as shown in Figure 6(a)) as well as high porosity caused by exaggerated grain growth during high-temperature sintering limit the performance of existing MOVs. Also shown in Figure 6, fine microstructures can be obtained for nanoenabled MOVs which can be sintered at reduced temperatures $\left(<1000^{\circ} \mathrm{C}\right)$. More varistor junctions in a unit MOV device can be provided so that higher voltage can be applied or smaller device can be fabricated for an equivalent voltage requirement.

Higher breakdown voltage $(>1 \mathrm{kV} / \mathrm{mm})$ and excellent electrical performance were achieved using the nanopowder precursors, new composition, and sintering profile. The electric field dependence of current for the MOV processed under different sintering conditions is shown in Figure 7. Not only the resistance of the ceramic composites decreases in a nonlinear way with an increase in the electric field, but also the transition field for the nonlinearity is pushed up by 10 times. This will enable miniaturization of MOVs and 


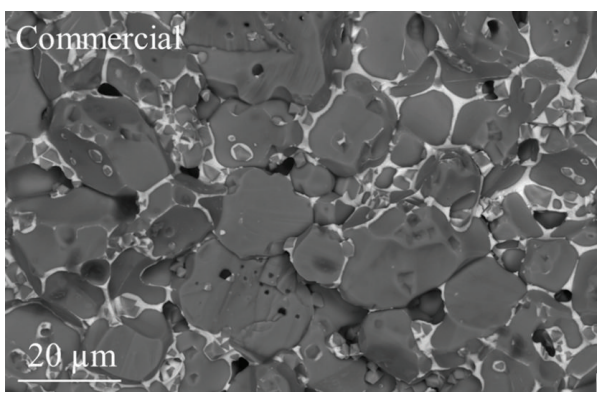

(a)

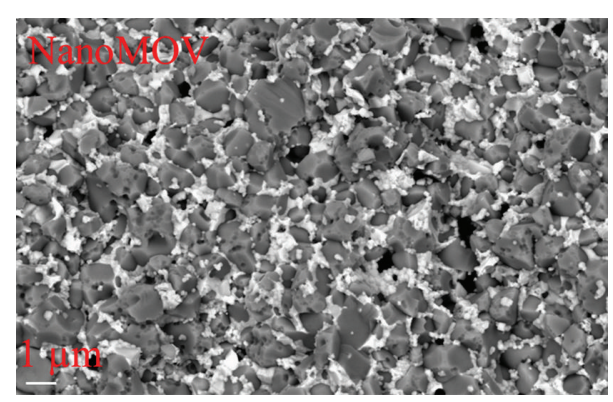

(b)

FIGURE 6: SEM images of microstructures of a commercial metal oxide varistor (a) and nanoenabled MOV sintered at $850^{\circ} \mathrm{C}(\mathrm{b})$.

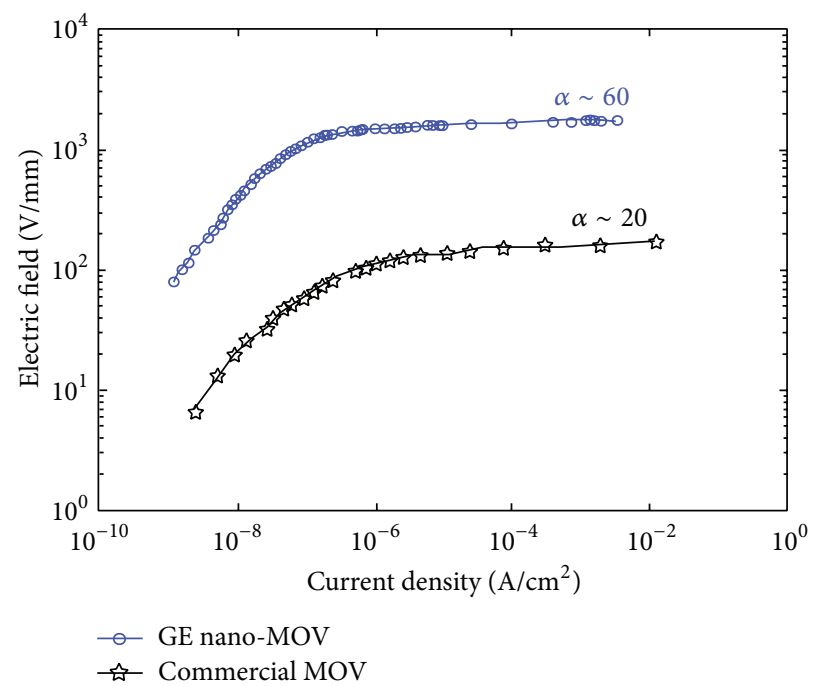

Figure 7: Nanoenabled MOVs with higher breakdown voltage after sintering at lower temperatures. The commercial MOVs made of coarse powder precursors were tested for comparison.

high voltage surge suppression. Besides, when the nano-sized metal oxide precursors were mixed with polymers to form composites, the similar $I-V$ characteristics can be observed. The field tuning behavior of the resistance can be fulfilled in the nanodielectric composites.

\section{Conclusions}

It is demonstrated that novel electrical/dielectric properties can be achieved via the nanodielectric engineering and interface engineering.

(1) Nanodielectric composites can be processed to host variety of ceramic particles and great particle dispersion and bonding with the host polymers can be fulfilled. The loading fraction and particle morphology, however, need to be controlled not to dramatically lower the dielectric strength.

(2) Electrically tunable composites were processed and a nonlinearly dependent dielectric constant was demonstrated by mixing polar nanoparticles with either low-K polyimide polymer or high-K PVDF polymers. The challenges are making the composite tunable at very low electric fields.
(3) High energy density $\left(15 \mathrm{~J} / \mathrm{cm}^{3}\right)$ can be achieved in nanodielectric composites containing nonlinear ferroelectric particles, with proper particle morphology, dispersion, and particle-polymer adhesion.

(4) $10 \mathrm{x}$ increase of voltage withstanding capability with low leakage current and higher nonlinearity can be realized in nanoenabled varistors. Refined and uniform submicron structures can be further explored in polymer based tunable resistors.

Further optimization of the performance of these electric device/component based such principles is undertaken.

\section{Conflict of Interests}

The authors declare that they have no conflict of interests regarding the publication of this paper.

\section{Acknowledgment}

Part of the work was sponsored by US Department of Defense under Contract of FA9451-08-C-0166. 


\section{References}

[1] A. S. Aricò, P. Bruce, B. Scrosati, J.-M. Tarascon, and W. Van Schalkwijk, "Nanostructured materials for advanced energy conversion and storage devices," Nature Materials, vol. 4, no. 5, pp. 366-377, 2005.

[2] A. Nourai, B. P. Martin, and D. R. Fitchett, "For new electricity storage technologies, the proof is in AEP's own tests," IEEE Power \& Energy Magzine, pp. 41-46, 2005.

[3] B. P. Roberts, "Growing use of large-scale power quality protection systems," in Proceedings of the Conference on Electrical Supply Industry, Shanghai, China, 2004.

[4] R. Baldick, "Reactive issues: reactive power in restructured markets," IEEE Power and Energy Magazine, vol. 2, no. 6, pp. 14-17, 2004.

[5] Q. Tan, P. Irwin, and Y. Cao, "Advanced dielectrics for capacitors," IEEJ Transactions on Fundamentals and Materials, vol. 126, no. 11, pp. 1153-1159, 2006.

[6] Y. Cao, P. C. Irwin, and K. Younsi, "The future of nanodielectrics in the electrical power industry," IEEE Transactions on Dielectrics and Electrical Insulation, vol. 11, no. 5, pp. 797-807, 2004.

[7] Y. Cao, S. Zhang, P. Irwin et al., GE TISCAT report 2010GRC431.

[8] D. Q. Tan, Q. Chen, Y. Cao, P. Irwin, and S. Heidger, "Polymer based nanodielectric composites," Presentation at EMA, Orlando, Fla, USA, 2010.

[9] Q. Tan, Y. Cao, and P. Irwin, "DC breakdown in polyetherimide composites and implication for structural engineering," in Proceedings of the International Conference on Solid Dielectrics (ICSD '07), pp. 411-414, Winchester, UK, July 2007.

[10] D. Q. Tan, K. Younsi, Y. Zhou, P. Irwin, and Y. Cao, "Nanoenabled metal oxide varistors," IEEE Transactions on Dielectrics and Electrical Insulation, vol. 16, no. 4, pp. 934-939, 2009. 

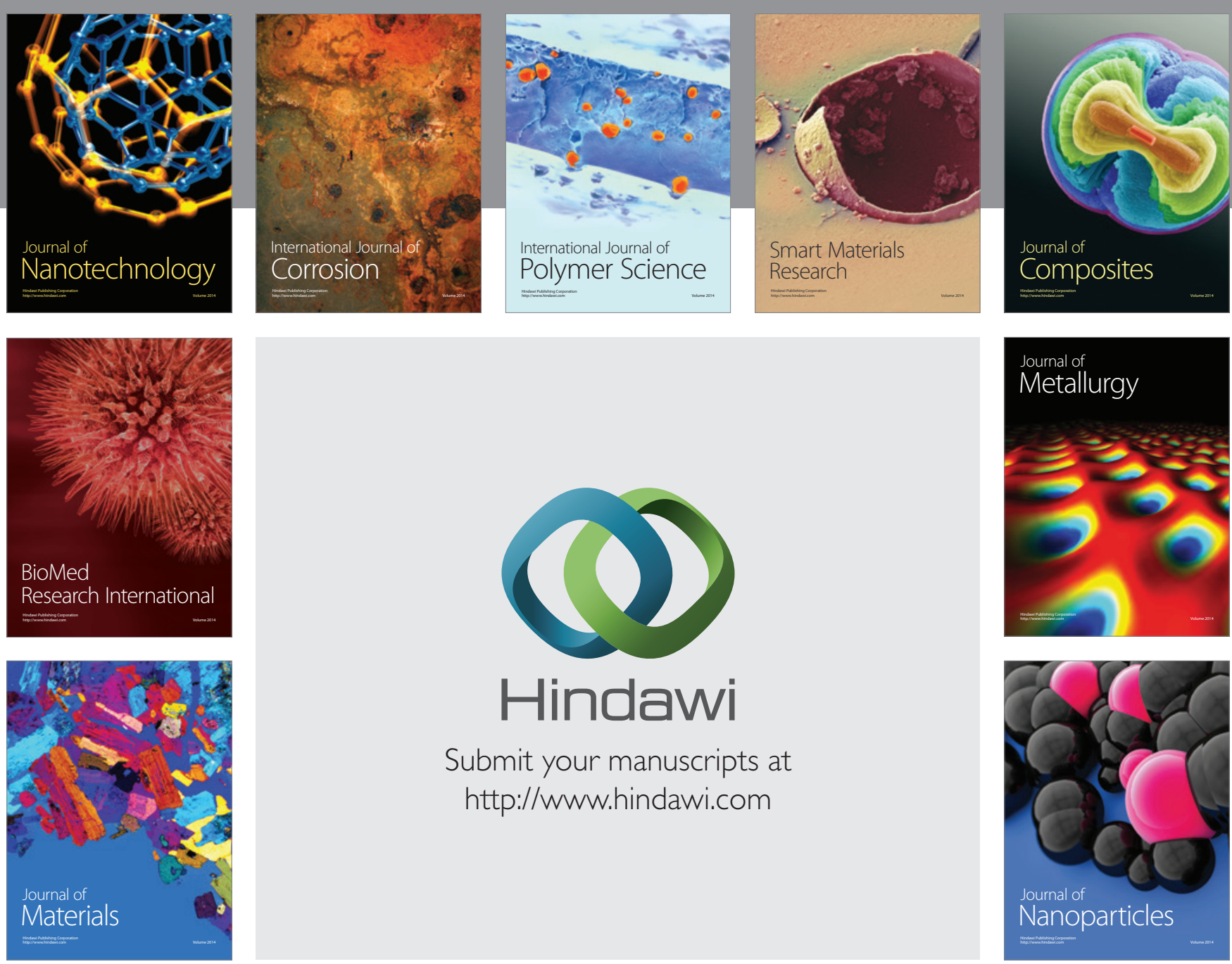

Submit your manuscripts at http://www.hindawi.com
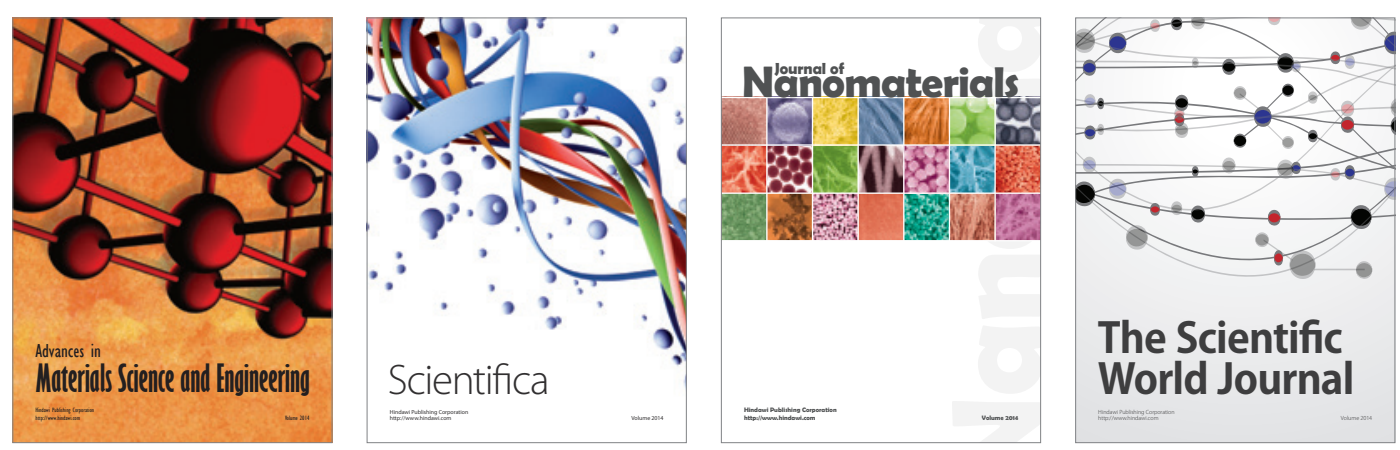

\section{The Scientific World Journal}
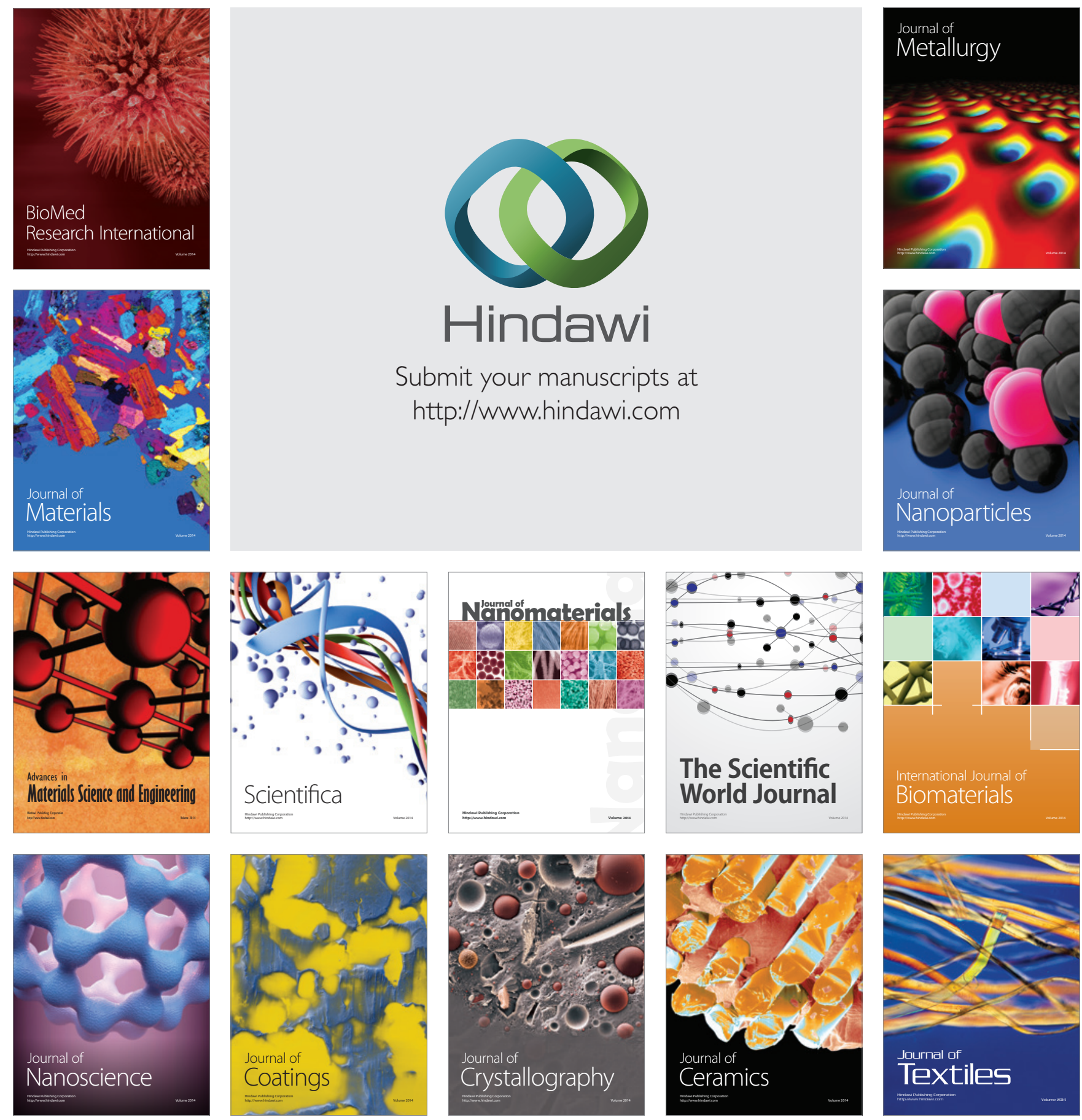